\title{
On Dependencies and Independencies of Fuzzy Implication Axioms
}

\author{
Y. Shi ${ }^{1}$, B. Van Gasse ${ }^{1}$, D. Ruan ${ }^{1,2}$, E.E. Kerre ${ }^{1}$ \\ ${ }^{1}$ Fuzziness and Uncertainty Modelling Research Unit, \\ Department of Applied Mathematics and Computer Science, \\ Ghent University, Krijgslaan 281 (S9), 9000 Gent, Belgium \\ ${ }^{2}$ Belgium Nuclear Research Centre (SCK•CEN), $2400 \mathrm{Mol}$, Belgium
}

\begin{abstract}
A fuzzy implication, commonly defined as a two-place operation on the unit interval, is an extension of the classical binary implication. It plays important roles in both mathematical and applied sides of fuzzy set theory. Besides the basic axioms, there are many potential fuzzy implication axioms, among which eight are widely used in the literature. Different fuzzy implications satisfying different subgroups of these eight axioms can be found. However, certain interrelationships exist between these eight axioms. But the results remain incomplete. This paper aims to lay bare the interrelationships between these eight axioms. The result is instrumental to propose a classification of fuzzy implications.
\end{abstract}

Key words: Fuzzy implication, fuzzy implication axioms, fuzzy logic operators, S-implication, R-implication

\section{Introduction}

One of the most important and interesting topics in fuzzy logic is to extend the classical binary logical operators conjunction, disjunction, negation and implication to fuzzy logic operators. The classical binary implication has the truth table 


\begin{tabular}{|c|c|c|}
\hline$p$ & $q$ & $p \rightarrow q$ \\
\hline 0 & 0 & 1 \\
\hline 0 & 1 & 1 \\
\hline 1 & 0 & 0 \\
\hline 1 & 1 & 1 \\
\hline
\end{tabular}

So the extension of the classical binary implication to the unit interval, a fuzzy implication, should be a $[0,1]^{2} \rightarrow[0,1]$ mapping $I$ that at least satisfies the boundary conditions:

$$
I(0,0)=I(0,1)=I(1,1)=1 \quad \text { and } \quad \mathrm{I}(1,0)=0 .
$$

Fuzzy implications play significant roles both in fuzzy logic and fuzzy set theory. A fuzzy implication can be used to determine

1. the truth value of a conditional rule. The conditional rule 'If $p$ then $q$ ' is defined by

$$
\operatorname{Tr}(p \Rightarrow q)=I(\operatorname{Tr}(p), \operatorname{Tr}(q))
$$

where $p$ and $q$ are two propositions and $\operatorname{Tr}(p)$ denotes the truth value of the proposition $p$.

2. a conditional relation between two linguistic variables $X$ and $Y$ on the universes of discourse $U$ and $V$, respectively. The rule 'If $X$ is $A$ then $Y$ is $B^{\prime}$ is defined by

$$
R_{X, Y}(u, v)=I(A(u), B(v)),
$$

where $R_{X, Y}$ denotes the conditional relation on $U \times V, A$ and $B$ are fuzzy sets on $U$ and $V$, respectively.

In the first aspect, a fuzzy implication is considered to be the extension of the implication in binary classical logic to the multivalued domain $[8,12]$. In the second aspect, a fuzzy implication is used in many applications as an operation between two fuzzy sets. For example, in the generalized modus ponens [12,15-17], in fuzzy subsethood measures [5], in fuzzy morphology operations $[11,13]$, and in determining the association rules in data mining [21].

There is no standard definition for a fuzzy implication as for a fuzzy conjunction, a fuzzy disjunction and a fuzzy negation. As the author of [10] states 'One of the main difficulties I have met during the preparation of lecture notes on some basic material concerning fuzzy set theory, consisted of a lack of standard definitions for basic elementary notions'. By taking into account the extensive literature about fuzzy implications $[3-5,8,9,20]$ we propose in this paper the following definition: 
Definition 1.1 A fuzzy implication $I$ is $a[0,1]^{2} \rightarrow[0,1]$ mapping that satisfies:

FI1. the first place antitonicity FA:

$\left(\forall\left(x_{1}, x_{2}, y\right) \in[0,1]^{3}\right)\left(x_{1}<x_{2} \Rightarrow I\left(x_{1}, y\right) \geq I\left(x_{2}, y\right)\right)$

FI2. the second place isotonicity SI:

$\left(\forall\left(x, y_{1}, y_{2}\right) \in[0,1]^{3}\right)\left(y_{1}<y_{2} \Rightarrow I\left(x, y_{1}\right) \leq I\left(x, y_{2}\right)\right)$

FI3. dominance of falsity of antecedent DF: $(\forall x \in[0,1])(I(0, x)=1)$;

FI4. dominance of truth of consequent DT: $(\forall x \in[0,1])(I(x, 1)=1)$;

FI5. boundary condition BC: $I(1,0)=0$.

Because of DF, DT and BC, a fuzzy implication $I$ satisfies the conditions in (1). There are several equivalent definitions for a fuzzy implication (as defined in Definition 1.1). For example, according to ([2], Lemma 1), the assumption that $I$ satisfies (1), FA and SI is an equivalent definition.

Many other potential axioms have been proposed in the literature devoted to fuzzy set theory, in order to obtain fuzzy implications fulfilling different requirements [3-5, $7,8,13,15,21,22]$, among which the most important ones are:

FI6. neutrality of truth NT: $(\forall x \in[0,1])(I(1, x)=x)$;

FI7. exchange principle EP: $\left(\forall(x, y, z) \in[0,1]^{3}\right)(I(x, I(y, z))=I(y, I(x, z)))$;

FI8. ordering principle OP: $\left(\forall(x, y) \in[0,1]^{2}\right)(I(x, y)=1 \Leftrightarrow x \leq y)$;

FI9. strong fuzzy negation principle SN: the mapping $N_{I}$ defined as $(\forall x \in$ $[0,1])\left(N_{I}(x)=I(x, 0)\right)$, is a strong fuzzy negation;

FI10. consequent boundary CB: $\left(\forall(x, y) \in[0,1]^{2}\right)(I(x, y) \geq y)$;

FI11. identity ID: $(\forall x \in[0,1])(I(x, x)=1)$;

FI12. contrapositive principle $(\mathrm{CP}):\left(\forall(x, y) \in[0,1]^{2}\right)(I(x, y)=I(N(y), N(x)))$, where $N$ is a strong fuzzy negation;

FI13. continuity CO: $I$ is a continuous mapping.

It is necessary to have a complete view of the interrelationships between these eight axioms NT-CO. On one hand, this helps to give a classification for all the fuzzy implications. On the other hand, this helps to solve some functional equations. Many works have studied the interrelationships between axioms NT-CO (e.g., $[1,3,4,8]$ ), but the complete interrelationship between these eight axioms remains missing. This paper aims to obtain the complete interrelationship between these axioms. Section 2 gives the necessary preliminaries. Section 3 gives the interrelationships between the axioms NT-CO. We provide each dependent case with a proof or citation, and each independent case with a counterexample. Section 4 summarizes the obtained results and illustrates the meanings. 


\section{Preliminaries}

Definition 2.1 ([4], Definition 0) A mapping $\varphi:[a, b] \rightarrow[a, b]([a, b] \subset \mathbb{R})$ is an order automorphism of the interval $[a, b]$ if it is continuous, strictly increasing and satisfies the boundary conditions: $\varphi(a)=a$ and $\varphi(b)=b$.

Definition 2.2 ([1], Definition 2) Two mappings $F, G:[0,1]^{n} \rightarrow[0,1], n \in \mathbb{N}$, are conjugate, if there exists an order automorphism $\varphi$ of the unit interval such that $G=F_{\varphi}$, where

$$
F_{\varphi}\left(x_{1}, x_{2}, \cdots, x_{n}\right)=\varphi^{-1}\left(F\left(\varphi\left(x_{1}\right), \varphi\left(x_{2}\right), \cdots, \varphi\left(x_{n}\right)\right)\right),
$$

Definition 2.3 A mapping $N:[0,1] \rightarrow[0,1]$ is a fuzzy negation if it is decreasing and satisfies: $N(0)=1, N(1)=0$. Moreover, if $N$ is involutive, i.e., $N(N(x))=x$, for all $x \in[0,1]$, then it is called a strong fuzzy negation.

The standard strong fuzzy negation $N_{0}$ is defined by

$$
(\forall x \in[0,1])\left(N_{0}(x)=1-x\right) .
$$

Any strong fuzzy negation $N$ is conjugate with the standard strong fuzzy negation $N_{0}$ [18].

Definition 2.4 A mapping $T:[0,1]^{2} \rightarrow[0,1]$ is a triangular norm (t-norm for short) if for all $x, y, z \in[0,1]$ it satisfies:

T1. boundary condition: $T(x, 1)=x$,

T2. isotonicity: $y \leq z$ implies $T(x, y) \leq T(x, z)$,

T3. commutativity: $T(x, y)=T(y, x)$,

T4. associativity: $T(x, T(y, z))=T(T(x, y), z)$.

Three important continuous t-norms are:

1. $T_{\mathbf{M}}(x, y)=\min (x, y), \quad$ (minimum)

2. $T_{\mathbf{P}}(x, y)=x y, \quad$ (product)

3. $T_{\mathbf{L}}(x, y)=\max (x+y-1,0)$. (Eukasiewicz)

Definition 2.5 A mapping $S:[0,1]^{2} \rightarrow[0,1]$ is a triangular conorm $(t$ conorm for short) if for all $x, y, z \in[0,1]$ it satisfies:

S1. boundary condition: $S(x, 0)=x$,

S2. isotonicity: $y \leq z$ implies $S(x, y) \leq S(x, z)$,

S3. commutativity: $S(x, y)=S(y, x)$,

S4. associativity: $S(x, S(y, z))=S(S(x, y), z)$. 
Three classes of fuzzy implications generated by t-norms, t-conorms and fuzzy negations are:

- S-implication: $I(x, y)=S(N(x), y)$,

- R-implication: $I(x, y)=\sup \{t \in[0,1] \mid T(x, t) \leq y\}$,

- QL-implication: $I(x, y)=S(N(x), T(x, y))$.

In addition to the above-mentioned three classes of fuzzy implications, we also consider in this paper other possible fuzzy implications that fulfill certain requirements.

\section{Getting FI6(NT) from the Other Axioms}

Theorem 3.1 ([3], Lemma 1.54(v), Corollary 1.57 (iii)) A fuzzy implication I satisfying $S N$ and $C P$ w.r.t. a strong fuzzy negation $N$ satisfies $N T$ iff $N_{I}=$ $N$.

In the rest of this section we consider the condition that $N_{I} \neq N$.

Proposition 3.2 ([1], Lemma 6) A fuzzy implication I satisfying EP and OP satisfies NT.

Proposition 3.3 ([3], Lemma 1.56(ii)) A fuzzy implication I satisfying EP and $S N$ satisfies $N T$.

Proposition 3.4 A fuzzy implication I satisfying EP and CO satisfies NT.

PROOF. Because $I$ satisfies EP, we have for all $x \in[0,1]$,

$$
I\left(1, N_{I}(x)\right)=I(1, I(x, 0))=I(x, I(1,0))=I(x, 0)=N_{I}(x) .
$$

Because $I$ is a continuous mapping, $N_{I}$ is a continuous mapping. Thus expression (3) is equivalent to $I(1, a)=a$, for all $a \in[0,1]$. Hence $I$ satisfies NT.

Remark 3.5 In Propositions 3.2, 3.3 and 3.4 we considered the following three cases:

$$
\begin{aligned}
& \mathrm{EP} \wedge \mathrm{OP} \Rightarrow \mathrm{NT} \\
& \mathrm{EP} \wedge \mathrm{SN} \Rightarrow \mathrm{NT} \\
& \mathrm{EP} \wedge \mathrm{CO} \Rightarrow \mathrm{NT}
\end{aligned}
$$

So we still need to consider the following two cases: 
$\mathrm{EP} \wedge \mathrm{CB} \wedge \mathrm{ID} \wedge \mathrm{CP} \stackrel{?}{\Rightarrow} \mathrm{NT}$

$\mathrm{OP} \wedge \mathrm{SN} \wedge \mathrm{CB} \wedge \mathrm{ID} \wedge \mathrm{CP} \wedge \mathrm{CO} \stackrel{?}{\Rightarrow} \mathrm{NT}$

Proposition 3.6 There exists a fuzzy implication I satisfying EP, CB, ID, $C P$ and not NT.

PROOF. The fuzzy implication $I_{1}$ stated in [6] is defined by

$$
I_{1}(x, y)=\left\{\begin{array}{ll}
0 & \text { if } x=1 \text { and } y=0 \\
1 & \text { else }
\end{array}, \quad x, y \in[0,1] .\right.
$$

Notice that $I_{1}$ is the greatest fuzzy implication. For all $x, y, z \in[0,1]$ we have

$$
\begin{aligned}
I_{1}\left(x, I_{1}(y, z)\right) & = \begin{cases}1, & \text { if } x<1 \text { or } y<1 \text { or } z>0 \\
0, & \text { else }\end{cases} \\
& =I_{1}\left(y, I_{1}(x, z)\right) .
\end{aligned}
$$

Therefore $I_{1}$ satisfies EP. Moreover, for all $x, y \in[0,1]$, we obtain:

$I_{1}(x, y) \geq y$,

$I_{1}(x, x)=1$,

$I_{1}(N(y), N(x))=I(x, y)$, for any strong fuzzy negation $N$.

Therefore $I_{1}$ satisfies $\mathrm{CB}$, ID and CP w.r.t. any strong fuzzy negation $N$. However, in case that $x \neq 1, I_{1}(1, x)=1 \neq x$. Therefore $I_{1}$ does not satisfy NT.

Proposition 3.7 There exists a fuzzy implication I satisfying OP, SN, CB, ID, CP, CO and not NT.

PROOF. Let a $[0,1]^{2} \rightarrow[0,1]$ mapping $I_{2}$ be defined by

$$
I_{2}(x, y)=\left\{\begin{array}{ll}
1 & \text { if } x \leq y \\
\sqrt{1-(x-y)^{2}} & \text { if } x>y
\end{array}, \quad x, y \in[0,1]\right.
$$

Checking that $I_{2}$ is a fuzzy implication is easy and therefore it is omitted. For all $x, y \in[0,1]$, we obtain:

$I_{2}(x, y)=1$ iff $x \leq y$,

$N_{I_{2}}(x)=I_{2}(x, 0)=\sqrt{1-x^{2}}=\varphi^{-1}(1-\varphi(x))$, where $\varphi(x)=x^{2}$ is an order automorphism of the unit interval,

$I_{2}(x, y) \geq y$, 
$I_{2}(x, x)=1$,

$I_{2}(1-y, 1-x)=I_{2}(x, y)$,

$I_{2}$ is a continuous mapping.

Therefore $I_{2}$ satisfies $\mathrm{OP}, \mathrm{SN}, \mathrm{CB}, \mathrm{ID}, \mathrm{CP}$ w.r.t. the standard strong fuzzy negation $N_{0}$, and CO. However, in case that $x \neq 1$ and $x \neq 0, I_{2}(1, x)=$ $\sqrt{2 x-x^{2}} \neq x$. Therefore $I_{2}$ does not satisfy NT.

So we considered all the possibilities that the fuzzy implication axiom NT can be implied from the other seven axioms. Moreover we stated for each independent case a counterexample.

\section{Getting FI7(EP) from the Other Axioms}

Proposition 4.1 There exists a fuzzy implication I satisfying NT, OP, SN, $C B, I D, C P, C O$ and not $E P$.

PROOF. Let a mapping $I_{3}$ be defined by

$$
I_{3}(x, y)=\left\{\begin{array}{ll}
1 & \text { if } x \leq y \\
1-(1-y+x y)(x-y) & \text { if } x>y
\end{array}, \quad x, y \in[0,1]\right.
$$

First we check that $I_{3}$ is a fuzzy implication. It is straightforward to check that $I_{3}(x, y) \in[0,1]$ for all $x, y \in[0,1]$. Moreover, for a fixed $y \in[0,1]$, let $0 \leq x_{1}<x_{2} \leq 1$. If $x_{1} \leq y$ then $I_{3}\left(x_{1}, y\right)=1 \geq I_{3}\left(x_{2}, y\right)$. If $y<x_{1}<x_{2} \leq 1$ then we have

$$
\begin{aligned}
& x_{1} y<x_{2} y \\
\Leftrightarrow & 1-y+x_{1} y<1-y+x_{2} y \\
\Leftrightarrow & \left(x_{1}-y\right)\left(1-y+x_{1} y\right)<\left(x_{2}-y\right)\left(1-y+x_{2} y\right) \\
\Leftrightarrow & I_{3}\left(x_{1}, y\right)>I_{3}\left(x_{2}, y\right) .
\end{aligned}
$$

Therefore $I_{3}$ satisfies FA.

Furthermore, for a fixed $x \in[0,1]$, let $0 \leq y_{1}<y_{2} \leq 1$. If $y_{2} \geq x$ then $I_{3}\left(x, y_{2}\right)=1 \geq I_{3}\left(x, y_{1}\right)$. If $0 \leq y_{1}<y_{2}<x$ then we have

$$
\begin{aligned}
& x y_{2}-x y_{1}<y_{2}-y_{1} \\
\Leftrightarrow & 1-y_{2}+x y_{2}<1-y_{1}+x y_{1} \\
\Leftrightarrow & \left(x-y_{2}\right)\left(1-y_{2}+x y_{2}\right)<\left(x-y_{1}\right)\left(1-y_{1}+x y_{1}\right) \\
\Leftrightarrow & I_{3}\left(x, y_{2}\right)>I_{3}\left(x, y_{1}\right) .
\end{aligned}
$$


Therefore $I_{3}$ also satisfies SI.

Checking that $I_{3}$ satisfies DF, DT and BC is easy and therefore it is omitted. Hence $I_{3}$ is a fuzzy implication.

For all $x, y \in[0,1]$, we obtain:

$$
\begin{aligned}
& I_{3}(1, x)=x, \\
& I_{3}(x, y)=1 \text { iff } x \leq y, \\
& N_{I_{3}}(x)=I_{3}(x, 0)=1-x, \\
& I_{3}(x, y) \geq y \\
& I_{3}(x, x)=1 \\
& I_{3}(1-y, 1-x)=I_{3}(x, y), \\
& I_{3} \text { is a continuous mapping. }
\end{aligned}
$$

Therefore $I_{3}$ satisfies $\mathrm{NT}, \mathrm{OP}, \mathrm{SN}, \mathrm{CB}, \mathrm{ID}, \mathrm{CP}$ w.r.t. the standard strong fuzzy negation $N_{0}$, and CO. However, take $x_{0}=0.3, y_{0}=0.9$ and $z_{0}=0.1$, we obtain $I\left(x_{0}, I\left(y_{0}, z_{0}\right)\right) \approx 0.9214$ and $I\left(y_{0}, I\left(x_{0}, z_{0}\right)\right) \approx 0.9210$. Therefore $I_{3}$ does not satisfy EP.

Remark 4.2 The fuzzy implication $I_{M M}$ presented in ([3], Table 1.5) is also an example that satisfies NT, OP, SN, CB, ID, CP w.r.t. the standard strong fuzzy negation $N_{0}$, and $\mathrm{CO}$ but not $\mathrm{EP}$. It is interesting to note that the implications $I_{3}$ and $I_{M M}$, despite satisfying exactly the same properties among FA-CO, are not conjugated to each other. We omit the proof here because it is rather technical.

$\mathrm{EP}$ is thus independent of any of the other seven axioms.

\section{Getting FI8(OP) from the Other Axioms}

Proposition 5.1 There exists a fuzzy implication I satisfying NT, EP, SN, $C B, I D, C P, C O$ and not $O P$.

PROOF. Given the strong fuzzy negation $N(x)=\sqrt{1-x^{2}}$, for all $x \in[0,1]$. The S-implication $I_{4}$ generated by the t-conorm $S_{\mathbf{L}}$ and the strong fuzzy negation $N$ is defined by

$$
I_{4}(x, y)=S_{\mathbf{L}}(N(x), y)=\min \left(\sqrt{1-x^{2}}+y, 1\right), x, y \in[0,1] .
$$

Because $I_{4}$ is an S-implication generated from a continuous t-conorm and a strong fuzzy negation, it satisfies NT, EP, SN, CB, CP w.r.t. the strong fuzzy negation $N$ and CO [8]. Moreover, for all $x, y \in[0,1], I_{4}(x, x)=1$. Therefore $I_{4}$ also satisfies ID. However, take $x_{0}=0.5$ and $y_{0}=0.4$, we obtain $I\left(x_{0}, y_{0}\right)=1$ while $x_{0}>y_{0}$. Therefore $I_{4}$ does not satisfy OP. 
Therefore OP is thus independent of any of the other seven axioms.

\section{Getting FI9(SN) from the Other Axioms}

Proposition 6.1 ([3], Lemma 1.5.4(v)) A fuzzy implication I satisfying NT and $C P$ w.r.t. a strong fuzzy negation $N$ satisfies $S N$. Moreover, $N_{I}=N$.

Corollary 6.2 A fuzzy implication I satisfying EP, OP and CP w.r.t. a strong fuzzy negation $N$ satisfies $S N$. Moreover, $N_{I}=N$.

PROOF. Straightforward from Propositions 3.2 and 6.1.

Corollary 6.3 A fuzzy implication I satisfying EP, CP w.r.t. a strong fuzzy negation $N$ and $C O$ satisfies $S N$. Moreover, $N_{I}=N$.

PROOF. Straightforward from Propositions 3.4 and 6.1.

Proposition 6.4 ([1], Lemma 14)([8], Corollary 1.1) A fuzzy implication I satisfying EP, OP and CO satisfies $S N$.

Remark 6.5 In Proposition 6.1, Corollary 6.2, Corollary 6.3 and Proposition 6.4 we considered the following four cases:

$$
\begin{aligned}
& \mathrm{NT} \wedge \mathrm{CP} \Rightarrow \mathrm{SN} \\
& \mathrm{EP} \wedge \mathrm{OP} \wedge \mathrm{CP} \Rightarrow \mathrm{SN} \\
& \mathrm{EP} \wedge \mathrm{OP} \wedge \mathrm{CO} \Rightarrow \mathrm{SN} \\
& \mathrm{EP} \wedge \mathrm{CP} \wedge \mathrm{CO} \Rightarrow \mathrm{SN}
\end{aligned}
$$

So we still need to consider the following five cases:

$$
\begin{aligned}
& \mathrm{NT} \wedge \mathrm{EP} \wedge \mathrm{OP} \wedge \mathrm{CB} \wedge \mathrm{ID} \stackrel{?}{\Rightarrow} \mathrm{SN} \\
& \mathrm{NT} \wedge \mathrm{EP} \wedge \mathrm{CB} \wedge \mathrm{ID} \wedge \mathrm{CO} \stackrel{?}{\Rightarrow} \mathrm{SN} \\
& \mathrm{NT} \wedge \mathrm{OP} \wedge \mathrm{CB} \wedge \mathrm{ID} \wedge \mathrm{CO} \stackrel{?}{\Rightarrow} \mathrm{SN} \\
& \mathrm{EP} \wedge \mathrm{CB} \wedge \mathrm{ID} \wedge \mathrm{CP} \stackrel{?}{\Rightarrow} \mathrm{SN} \\
& \mathrm{OP} \wedge \mathrm{CB} \wedge \mathrm{ID} \wedge \mathrm{CP} \wedge \mathrm{CO} \stackrel{?}{\Rightarrow} \mathrm{SN}
\end{aligned}
$$

Proposition 6.6 ([8], Table 1.1) There exists a fuzzy implication satisfying $N T, E P, O P, C B, I D$ and not $S N$. 
PROOF. The Gödel implication

$$
I_{G}(x, y)=\left\{\begin{array}{ll}
1, & \text { if } x \leq y \\
y, & \text { if } x>y
\end{array}, \quad x, y \in[0,1]\right.
$$

is an R-implication generated by the continuous t-norm $T_{\mathbf{M}}$. Therefore $I_{G}$ satisfies NT, EP, OP, CB and ID [8]. However we have for all $x \in[0,1]$,

$$
N_{I_{G}}(x)=I_{G}(x, 0)= \begin{cases}1, & \text { if } x=0 \\ 0, & \text { if } x>0\end{cases}
$$

Therefore $I_{G}$ does not satisfy SN.

Proposition 6.7 There exists a fuzzy implication I satisfying NT, EP, CB, $I D, C O$ and not $S N$.

PROOF. Given the fuzzy negation $N(x)=1-x^{2}$, for all $x \in[0,1]$. The S-implication generated from the t-conorm $S_{\mathbf{L}}$ and the fuzzy negation $N$ is defined by

$$
I_{5}(x, y)=\min \left(1-x^{2}+y, 1\right) \quad x, y \in[0,1] .
$$

For all $x, y \in[0,1]$, we obtain:

$$
\begin{aligned}
& I_{5}(1, x)=x \\
& I_{5}(x, y) \geq y \\
& I_{5}(x, x)=1 \\
& I_{5} \text { is a continuous mapping. }
\end{aligned}
$$

Therefore $I_{5}$ satisfies NT, CB, ID and CO. Moreover, because $I_{5}$ is an $(S, N)$ implication generated from the Eukasiewicz t-conorm and the strict fuzzy negation $N(x)=1-x^{2}$, it then also satisfies EP ([3], Proposition 2.4.3(i)). However, we have for all $x \in[0,1]$

$$
N_{I_{5}}(x)=I_{5}(x, 0)=1-x^{2}
$$

which is not a strong fuzzy negation. Therefore $I_{5}$ does not satisfy SN.

Proposition 6.8 There exists a fuzzy implication I satisfying NT, OP, CB, $I D, C O$ and not $S N$. 
PROOF. Let a mapping $I_{6}$ be defined by

$$
I_{6}(x, y)=\left\{\begin{array}{l}
1, \quad \text { if } x \leq y \\
\frac{y}{1+\sqrt{1-x}}+\sqrt{1-x}, \quad \text { if } x>y
\end{array}, \quad x, y \in[0,1] .\right.
$$

First we show that $I_{6}$ is a fuzzy implication. It is straightforward to check that $I_{6}(x, y) \in[0,1]$ for all $x, y \in[0,1]$. Moreover, $I_{6}$ satisfying SI-BC is trivial. We prove that $I_{6}$ also satisfies FA. Indeed, for all $0 \leq y<x \leq 1$ we obtain

$$
\frac{\partial I_{6}(x, y)}{\partial x}=\frac{-1}{2}\left(1-\frac{y}{(1+\sqrt{1-x})^{2}}\right) \frac{1}{\sqrt{1-x}},
$$

and

$$
\begin{aligned}
& y<x<2-x+2 \sqrt{1-x} \\
\Rightarrow & y<(1+\sqrt{1-x})^{2} \\
\Rightarrow & \left(1-\frac{y}{(1+\sqrt{1-x})^{2}}\right) \frac{1}{\sqrt{1-x}}>0 \\
\Rightarrow & \frac{\partial I_{6}(x, y)}{\partial x}<0 .
\end{aligned}
$$

Furthermore, for all $0 \leq x \leq y \leq 1, \frac{\partial I_{6}(x, y)}{\partial x}=0$. Thus $\frac{\partial I_{6}(x, y)}{\partial x} \leq 0$, for all $x$, $y \in[0,1]$. Therefore $I_{6}$ satisfies FA.

Next we show that $I_{6}$ satisfies NT, OP, CB, ID and CO but not SN.

Indeed, for all $x, y \in[0,1]$

$$
\begin{aligned}
& I_{6}(1, x)=x \\
& I_{6}(x, y)=1 \text { iff } x \leq y, \\
& I_{6}(x, y) \geq y
\end{aligned}
$$

$I_{6}$ is a continuous mapping.

Therefore $I_{6}$ satisfies NT, OP, CB and CO. However, we have for all $x \in[0,1]$

$$
N_{I_{6}}(x)=I_{6}(x, 0)=\sqrt{1-x}
$$

which is not a strong fuzzy negation. Therefore $I_{6}$ does not satisfy SN

Proposition 6.9 There exists a fuzzy implication I satisfying EP, CB, ID, $C P$ and not $S N$.

The fuzzy implication $I_{1}$ stated in the proof of Proposition 3.6 satisfies EP, $\mathrm{CB}$, ID and $\mathrm{CP}$ w.r.t. any strong fuzzy negation $N$. However, we have

$$
N_{I_{1}}(x)=I_{1}(x, 0)=\left\{\begin{array}{ll}
1, & \text { if } x<1 \\
0, & \text { if } x=1
\end{array}, \quad x \in[0,1],\right.
$$


which is not a strong fuzzy negation. Therefore $I_{1}$ does not satisfy SN.

Proposition 6.10 There exists a fuzzy implication I satisfying $O P, C B, I D$, $C P, C O$ and not $S N$.

PROOF. Let a $[0,1]^{2} \rightarrow[0,1]$ mapping $I_{7}$ be defined by

$$
I_{7}(x, y)=\left\{\begin{array}{l}
1, \quad \text { if } x \leq y \\
\sqrt{1-(x-y),} \quad \text { if } x>y
\end{array}, \quad x, y \in[0,1] .\right.
$$

Checking that $I_{7}$ is a fuzzy implication is easy and therefore it is omitted. For all $x, y \in[0,1]$, we obtain:

$$
\begin{aligned}
& I_{7}(x, y)=1 \text { iff } x \leq y, \\
& I_{7}(x, y) \geq y \\
& I_{7}(x, x)=1 \\
& I_{7}(1-y, 1-x)=I_{7}(x, y),
\end{aligned}
$$

$I_{7}$ is a continuous mapping.

Therefore $I_{7}$ satisfies $\mathrm{OP}, \mathrm{CB}, \mathrm{ID}, \mathrm{CP}$ w.r.t. the standard strong fuzzy negation $N_{0}$, and CO. However, we have for all $x \in[0,1]$

$$
N_{I_{7}}(x)=I_{7}(x, 0)=\sqrt{1-x},
$$

which is not a strong fuzzy negation. Therefore $I_{7}$ does not satisfy SN.

Remark 6.11 The fuzzy implication $I_{B Z}$ presented in ([3], Example 1.5.10(iv)) also satisfies $\mathrm{OP}, \mathrm{CB}$, ID, CP w.r.t. the standard strong fuzzy negation $N_{0}$, and $\mathrm{CO}$ but not $\mathrm{SN}$. It is interesting to note that the two implications $I_{7}$ and $I_{B Z}$, despite satisfying exactly the same properties among FA-CO, are not conjugated to each other. Indeed, $I_{7}(x, 0)$ is strictly decreasing while $I_{B Z}(x, 0)$ is not.

So we considered all the possibilities that the fuzzy implication axiom SN can be implied from the other seven axioms. Moreover we stated for each independent case a counterexample.

\section{Getting FI10(CB) from the Other Axioms}

Proposition 7.1 ([4],Lemma 1 (viii)) A fuzzy implication I satisfying NT satisfies $C B$.

Corollary 7.2 A fuzzy implication I satisfying EP and SN satisfies CB. 
PROOF. Straightforward from Propositions 3.3 and 7.1.

Corollary 7.3 A fuzzy implication I satisfying EP and CO satisfies CB.

PROOF. Straightforward from Propositions 3.4 and 7.1.

Proposition 7.4 ([1],Lemma 6) A fuzzy implication I satisfying EP and OP satisfies $C B$.

Remark 7.5 In Proposition 7.1, Corollary 7.2, Corollary 7.3 and Proposition 7.4 we considered the following four cases:

$$
\begin{aligned}
& \mathrm{NT} \Rightarrow \mathrm{CB} \\
& \mathrm{EP} \wedge \mathrm{OP} \Rightarrow \mathrm{CB} \\
& \mathrm{EP} \wedge \mathrm{SN} \Rightarrow \mathrm{CB} \\
& \mathrm{EP} \wedge \mathrm{CO} \Rightarrow \mathrm{CB}
\end{aligned}
$$

So we still need to consider the following two cases:

$$
\begin{aligned}
& \mathrm{EP} \wedge \mathrm{ID} \wedge \mathrm{CP} \stackrel{?}{\Rightarrow} \mathrm{CB} \\
& \mathrm{OP} \wedge \mathrm{SN} \wedge \mathrm{ID} \wedge \mathrm{CP} \wedge \mathrm{CO} \stackrel{?}{\Rightarrow} \mathrm{CB}
\end{aligned}
$$

Proposition 7.6 There exists a fuzzy implication I satisfying EP, ID, CP and not $C B$.

PROOF. Let a fuzzy implication $I_{8}$ be defined by

$$
I_{8}(x, y)=\left\{\begin{array}{ll}
1, & \text { if } x \leq 0.5 \text { or } y \geq 0.5 \\
0, & \text { else }
\end{array}, \quad x, y \in[0,1]\right.
$$

For all $x, y, z \in[0,1]$ we obtain

$$
\begin{aligned}
I_{8}\left(x, I_{8}(y, z)\right) & = \begin{cases}1, & \text { if } x \leq 0.5 \text { or } y \leq 0.5 \text { or } z \geq 0.5 \\
0, & \text { else }\end{cases} \\
& =I_{8}\left(y, I_{8}(x, z)\right)
\end{aligned}
$$

Therefore $I_{8}$ satisfies EP. Moreover, for all $x, y \in[0,1]$, we obtain:

$$
\begin{aligned}
& I_{8}(x, x)=1, \\
& I_{8}(1-y, 1-x)=I_{8}(x, y) .
\end{aligned}
$$


Therefore $I_{8}$ satisfies ID and CP w.r.t. the standard strong fuzzy negation $N_{0}$. However, take $x_{0}=1$ and $y_{0}=0.1$, we obtain $I_{8}\left(x_{0}, y_{0}\right)=0<y_{0}$. Therefore $I_{8}$ does not satisfy $\mathrm{CB}$.

Proposition 7.7 There exists a fuzzy implication I satisfying OP, SN, ID, $C P, C O$ and not $C B$.

PROOF. Let a $[0,1]^{2} \rightarrow[0,1]$ mapping $I_{9}$ be represented as

$$
I_{9}(x, y)=\left\{\begin{array}{l}
1, \quad \text { if } x \leq y \\
(1-\sqrt{x-y})^{2}, \quad \text { if } x>y
\end{array}, \quad x, y \in[0,1]\right.
$$

Checking that $I_{9}$ is a fuzzy implication is easy and therefore it is omitted. For all $x, y \in[0,1]$, we obtain:

$$
I_{9}(x, y)=1 \text { iff } x \leq y
$$

$N_{I_{9}}(x)=I_{9}(x, 0)=(1-\sqrt{x})^{2}=\varphi^{-1}(1-\varphi(x))$, where $\varphi(x)=\sqrt{x}$ is an order automorphism of the unit interval,

$I_{9}(x, x)=1$,

$I_{9}(1-y, 1-x)=I_{9}(x, y)$,

$I_{9}$ is a continuous mapping.

Therefore $I_{9}$ satisfies OP, SN, ID, CP w.r.t. the standard strong fuzzy negation $N_{0}$, and CO. However, take $x_{0}=1$ and $y_{0}=0.64$, we obtain $I_{9}\left(x_{0}, y_{0}\right)=0.16<$ $y_{0}$. Therefore $I_{9}$ does not satisfy CB.

So we considered all the possibilities that the fuzzy implication axiom CB can be implied from the other seven axioms. Moreover we stated for each independent case a counterexample.

\section{Getting FI11(ID) from the Other Axioms}

Proposition 8.1 A fuzzy implication I satisfying OP satisfies ID.

PROOF. Straightforward.

Remark 8.2 In Proposition 8.1 we considered the following case:

$$
\mathrm{OP} \Rightarrow \mathrm{ID}
$$


So we still need to consider the following case:

$\mathrm{NT} \wedge \mathrm{EP} \wedge \mathrm{SN} \wedge \mathrm{CB} \wedge \mathrm{CP} \wedge \mathrm{CO} \stackrel{?}{\Rightarrow} \mathrm{ID}$

Proposition 8.3 There exists a fuzzy implication I satisfying NT, EP, SN, $C B, C P, C O$ and not ID.

PROOF. The Kleene-Dienes implication $I_{K D}(x, y)=\max (1-x, y)$, for all $(x, y) \in[0,1]^{2}$ is an S-implication generated from the t-conorm $S_{\mathrm{M}}$ and the standard strong fuzzy negation $N_{0}$. Therefore $I_{K D}$ satisfies NT, EP, SN, CB, $\mathrm{CP}$ w.r.t. the standard strong fuzzy negation $N_{0}$, and CO. However, for $x_{0}=$ 0.1 , we obtain $I_{K D}\left(x_{0}, x_{0}\right)=0.9 \neq 1$. Therefore $I_{K D}$ does not satisfy ID.

So we considered all the possibilities that the fuzzy implication axiom ID can be implied from the other seven axioms, and stated for the independent case a counterexample.

\section{Getting FI12(CP) from the Other Axioms}

Proposition 9.1 ([4],Lemma 1(ix)) A fuzzy implication I satisfying EP and $S N$ satisfies CP w.r.t. the strong fuzzy negation $N_{I}$.

Proposition 9.2 ([1]) A fuzzy implication I satisfying EP, OP and CO satisfies CP w.r.t. the strong fuzzy negation $N_{I}$.

Remark 9.3 In Propositions 9.1 and 9.2 we considered the following two cases:

$$
\begin{aligned}
& \mathrm{EP} \wedge \mathrm{SN} \Rightarrow \mathrm{CP} \\
& \mathrm{EP} \wedge \mathrm{OP} \wedge \mathrm{CO} \Rightarrow \mathrm{CP}
\end{aligned}
$$

So we still need to consider the following three cases:

$$
\begin{aligned}
& \mathrm{NT} \wedge \mathrm{EP} \wedge \mathrm{OP} \wedge \mathrm{CB} \wedge \mathrm{ID} \stackrel{?}{\Rightarrow} \mathrm{CP} \\
& \mathrm{NT} \wedge \mathrm{EP} \wedge \mathrm{CB} \wedge \mathrm{ID} \wedge \mathrm{CO} \stackrel{?}{\Rightarrow} \mathrm{CP} \\
& \mathrm{NT} \wedge \mathrm{OP} \wedge \mathrm{SN} \wedge \mathrm{CB} \wedge \mathrm{ID} \wedge \mathrm{CO} \stackrel{?}{\Rightarrow} \mathrm{CP}
\end{aligned}
$$

Proposition 9.4 There exists a fuzzy implication I satisfying NT, EP, OP, $C B, I D$ and not $C P$. 
According to the proof of Proposition 6.6, the Gödel implication $I_{G}$ satisfies $\mathrm{NT}, \mathrm{EP}, \mathrm{OP}, \mathrm{CB}$ and ID. However, for any strong fuzzy negation $N$ we obtain

$$
I_{G}(N(y), N(x))=\left\{\begin{array}{l}
1, \quad \text { if } x \leq y \\
N(x), \quad \text { if } x>y
\end{array}\right.
$$

In case that $x>y$ and $N(x) \neq y, I_{G}(N(y), N(x)) \neq I_{G}(x, y)$. Therefore $I_{G}$ does not satisfy $\mathrm{CP}$ w.r.t. any strong fuzzy negation.

Proposition 9.5 There exists a fuzzy implication I satisfying NT, EP, CB, ID, $C O$ and not $C P$.

The fuzzy implication $I_{5}$ stated in the proof of Proposition 6.7 satisfies NT, $\mathrm{EP}, \mathrm{CB}$, ID and CO. However, because for all $x \in[0,1], N_{I_{5}}(x)=1-x^{2}$, which is not a strong fuzzy negation, according to Corollary 1.5 .5 in [3], $I_{5}$ does not satisfy CP w.r.t. any strong fuzzy negation.

Proposition 9.6 There exists a fuzzy implication I satisfying NT, OP, SN, $C B, I D, C O$ and not $C P$.

PROOF. Let a mapping $I_{10}$ be defined by

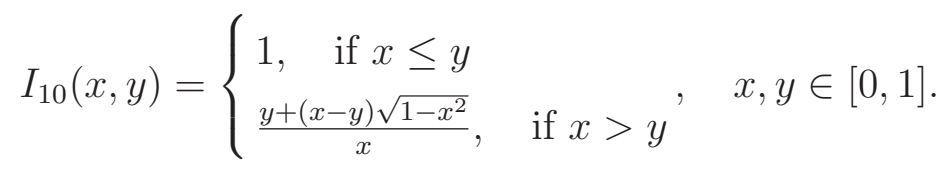

First we check that $I_{10}$ is a fuzzy implication. It is straightforward to check that $I_{10}(x, y) \in[0,1]$ for all $x, y \in[0,1]$. Moreover, for a fixed $y \in[0,1]$, let $0 \leq x_{1}<x_{2} \leq 1$. If $x_{1} \leq y$ then $I_{10}\left(x_{1}, y\right)=1 \geq I_{10}\left(x_{2}, y\right)$. If $y<x_{1}<x_{2} \leq 1$ then define a mapping $\left.\left.f_{y}:\right] y, 1\right] \rightarrow[0,1]$ as $f_{y}(x)=\frac{y+(x-y) \sqrt{1-x^{2}}}{x}$, for all $x \in$ ] $y, 1]$. We have

$$
\frac{d f_{y}}{d x}=\frac{y-x}{\sqrt{1-x^{2}}}-\frac{y\left(1-\sqrt{1-x^{2}}\right)}{x^{2}}<0 .
$$

Thus $f_{y}$ is a decreasing mapping. Therefore $I_{10}\left(x_{1}, y\right)>I_{10}\left(x_{2}, y\right)$. Hence $I_{10}$ satisfies FA.

Furthermore, for a fixed $x \in[0,1]$, let $0 \leq y_{1}<y_{2} \leq 1$. If $y_{2} \geq x$ then $I_{10}\left(x, y_{2}\right)=1 \geq I_{10}\left(x, y_{1}\right)$. If $0 \leq y_{1}<y_{2}<x$ then we have

$$
\begin{aligned}
& I_{10}\left(x, y_{2}\right)-I_{10}\left(x, y_{1}\right) \\
= & \frac{\left(y_{2}-y_{1}\right)\left(1-\sqrt{1-x^{2}}\right)}{x}>0
\end{aligned}
$$

Therefore $I_{10}$ also satisfies SI.

Checking that $I_{10}$ satisfies DF, DT and BC is easy and therefore it is omitted. 
Hence $I_{10}$ is a fuzzy fuzzy imlication.

For all $x, y \in[0,1]$, we obtain:

$$
\begin{aligned}
& I_{10}(1, x)=x, \\
& I_{10}(x, y)=1 \text { iff } x \leq y, \\
& N_{I_{10}}(x)=I_{10}(x, 0)=\sqrt{1-x^{2}}=\varphi^{-1}(1-\varphi(x)) \text {, where } \varphi(x)=x^{2} \text { is an order } \\
& \text { automorphism of the unit interval, } \\
& I_{10}(x, x)=1, \\
& I_{10} \text { is a continuous mapping. }
\end{aligned}
$$

Therefore $I_{10}$ satisfies $\mathrm{NT}, \mathrm{OP}, \mathrm{SN}, \mathrm{ID}$ and $\mathrm{CO}$. If $I_{10}$ satisfies $\mathrm{CP}$ w.r.t. a strong fuzzy negation $N$, then for all $x \in[0,1]$, we obtain

$$
N(x)=I_{10}(1, N(x))=I_{10}(x, 0)=N_{I_{10}}(x)=\sqrt{1-x^{2}} .
$$

However, take $x_{0}=0.8$ and $y_{0}=0.1$, we obtain $I_{10}\left(x_{0}, y_{0}\right)=0.65$ and $I_{10}\left(N\left(y_{0}\right), N\left(x_{0}\right)\right) \approx 0.643$. Therefore $I_{10}$ does not satisfy CP w.r.t. any strong fuzzy negation $N$.

So we considered all the possibilities that the fuzzy implication axiom CP can be implied from the other seven axioms. Moreover we stated for each independent case a counterexample.

\section{Getting FI13(CO) from the Other Axioms}

Proposition 10.1 There exists a fuzzy implication I satisfying NT, EP, OP, $S N, C B, I D, C P$ and not $C O$.

PROOF. Let $N$ be a strong fuzzy negation. Recall the $R_{0}$-implication stated in [14] which is defined by

$$
\left(I_{\min _{0}}\right)_{N}(x, y)=\left\{\begin{array}{l}
1, \quad \text { if } x \leq y \\
\max (N(x), y), \quad \text { if } x>y
\end{array}, \quad x, y \in[0,1]\right.
$$

$\left(I_{\text {min }_{0}}\right)_{N}$ is the R-implication generated by the left-continuous t-norm, nilpotent minimum [7]:

$$
\left(T_{\min _{0}}\right)_{N}(x, y)=\left\{\begin{array}{l}
\min (x, y), \quad \text { if } y>N(x) \\
0, \quad \text { if } y \leq N(x)
\end{array}, \quad x, y \in[0,1]\right.
$$


$\left(I_{\text {min }_{0}}\right)_{N}$ satisfies $\mathrm{NT}, \mathrm{EP}, \mathrm{OP}, \mathrm{SN}, \mathrm{CB}, \mathrm{ID}$ and $\mathrm{CP}$ w.r.t. $N$, and is rightcontinuous in the second place [14] but it is not continuous.

Therefore $\mathrm{CO}$ is independent of any of the other seven axioms.

\section{Summary}

From Sections 4, 5 and 10 the three axioms EP, OP and CO are essential because they are totally independent of the other axioms. On the other hand, they are really important because the combination of them can imply all the other five axioms. From Section 8, the axiom ID is relatively essential because only OP can imply it. The combination of the other six axioms cannot imply ID. However, none of the other axioms is dependent on ID.

Table 1 summarizes the results we obtained in Sections 3-10.

Let $S_{1}$ denote a subset of A defined by

$$
A=\{\mathrm{NT}, \mathrm{EP}, \mathrm{OP}, \mathrm{SN}, \mathrm{CB}, \mathrm{ID}, \mathrm{CP}, \mathrm{CO}\}
$$

and

$$
S_{2}=A-S_{1}
$$

Then from Table 1 we can judge if a fuzzy implication satisfies all the axioms in $S_{1}$ then it also satisfies the axioms of $S_{2}$. For example, let

$$
S_{1}=\{\mathrm{EP}, \mathrm{SN}, \mathrm{ID}\}
$$

Then

$$
S_{2}=\{\mathrm{NT}, \mathrm{OP}, \mathrm{CB}, \mathrm{CP}, \mathrm{CO}\} .
$$

According to rows 2, 10 and 14 of Table 1 , the fuzzy implication $I_{4}$ and $I_{\min _{0}}$ of Table 2, we obtain:

$$
S_{1} \Rightarrow\{\mathrm{NT}, \mathrm{CB}, \mathrm{CP}\}
$$

Finally we summarize all the examples in Table 2, where ' $\mathrm{Y}$ ' denotes 'yes' and ' $N$ ' denotes 'no'.

Acknowledgement The authors want to thank the referees for their helpful comments that substantially improved this paper.

Bart Van Gasse would like to thank the Research Foundation-Flanders for funding his research. 


\section{References}

[1] M. Baczyński, Residual implications revisited. Notes on the Smets-Magrez Theorem, Fuzzy Sets and Systems 145 (2004) 267-277.

[2] M. Baczyński, J. Drewniak, Monotonic fuzzy implications, in: P.S. Szczepaniak, P.J.G. Lisboa, J. Kacprzyk (Eds.), Fuzzy Systems in Medicine, pp. 90-111, Physica, Heidelberg, 2000.

[3] M. Baczyński, B. Jayaram, Fuzzy Implications, Springer-Verlag, Berlin Heidelberg, 2008.

[4] H. Bustince, P. Burillo, F. Soria, Automorphisms, negations and implication operators, Fuzzy Sets and Systems 134 (2003) 209-229.

[5] H. Bustince, M. Pagola, E. Barrenechea, Construction of fuzzy indices from fuzzy DI-subsethood measures: Application to the global comparison of images, Information Sciences 177 (2007) 906-929.

[6] J. Drewniak, Invariant fuzzy implications, Soft Computing 10 (2006) 506-513.

[7] J.C. Fodor, Contrapositive symmetry of fuzzy implications, Fuzzy Sets and Systems 69 (1995) 141-156.

[8] J.C. Fodor, M. Roubens, Fuzzy Preference Modelling and Multicriteria Decision Support, Kluwer Academic Publishers, Dordrecht, 1994.

[9] B. Jayaram, Rule reduction for efficient inferencing in similarity based reasoning, International Journal of Approximate Reasoning 48 (2008) 156-173.

[10] E.E. Kerre, A call for crispness in fuzzy set theory, Fuzzy Sets and Systems 29 (1989) 57-65.

[11] E.E. Kerre, M. Nachtegael, Fuzzy Techniques in Image Processing, PhysicaVerlag, New York, 2000.

[12] G.J. Klir, B. Yuan, Fuzzy Sets and Fuzzy Logic, Theory and Applications, Prentice Hall, New Jersey, 1995.

[13] M. Nachtegael, H. Heijmans, D. Van der Weken, E. Kerre, Fuzzy Adjunctions in Mathematical Morphology, Proc. of JCIS 2003, North Carolina, USA, sept, (2003) 202-205.

[14] D. Pei, $R_{0}$ implication: characteristics and applications, Fuzzy Sets and Systems 131 (2002) 297-302.

[15] D. Ruan, E.E. Kerre, Fuzzy implication operators and generalized fuzzy method of cases, Fuzzy Sets and Systems 54 (1993) 23-37.

[16] D. Ruan, E.E. Kerre, Fuzzy IF-THEN Rules in Computational Intelligence: Theory and Applications, Kluwer Academic Publishers, Boston, 2000. 
[17] M. Mas, M. Monserrat, J. Torrens, E. Trillas, A survey on fuzzy implication functions, IEEE Transactions on Fuzzy Systems 15(6) (2007) 1107-1121.

[18] E. Trillas, sobre funciones de negación en la teoría de conjuntos difusos, Stochastica 3(1) (1979) 47-60.

[19] E. Trillas, C. Alsina, E. Renedo, A. Pradera, On contra-symmetry and MPT conditionality in fuzzy logic, International Journal of Intelligent Systems 20 (2005) 313-326.

[20] R.R. Yager, On some new classes of implication operators and their role in approximate reasoning, Information Sciences 167 (2004) 193-216.

[21] P. Yan, G. Chen, Discovering a cover set of ARsi with hierarchy from quantitative databases, Information Sciences 173 (2005) 319-336.

[22] H.Y. Zhang, W.X. Zhang, Hybrid monotonic inclusion measure and its use in measuring similarity and distance between fuzzy sets, Fuzzy Sets and Systems 160 (2009) 107-118. 


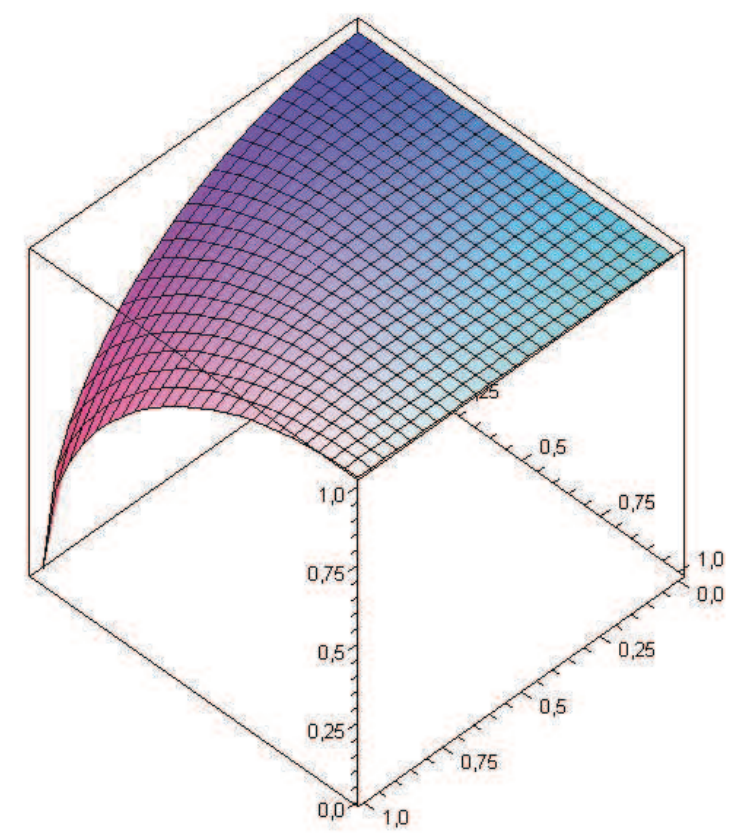

Fig. 1. The fuzzy implication $I_{2}$ in the proof of Proposition 3.7

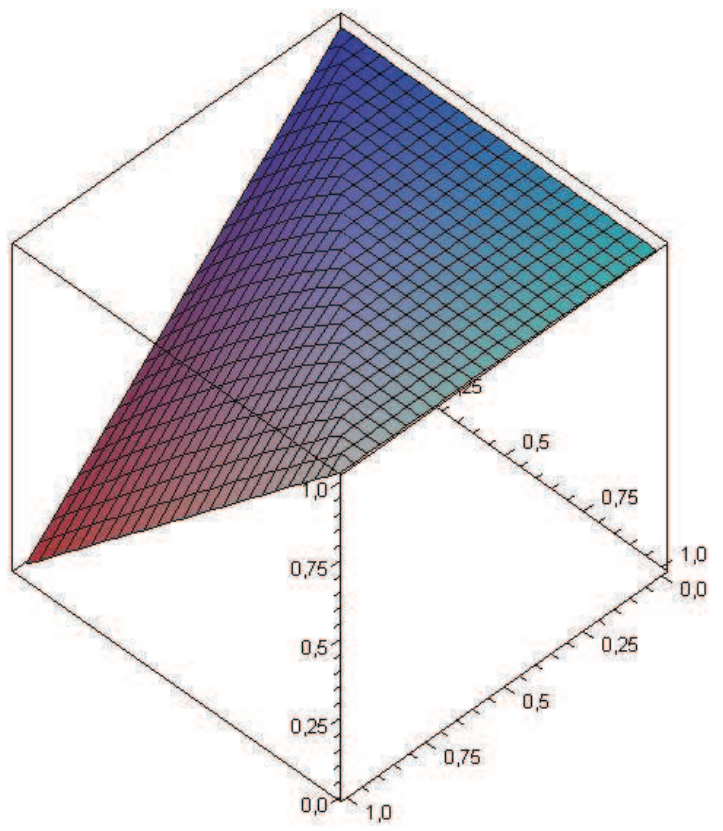

Fig. 2. The fuzzy implication $I_{3}$ in the proof of Proposition 4.1 


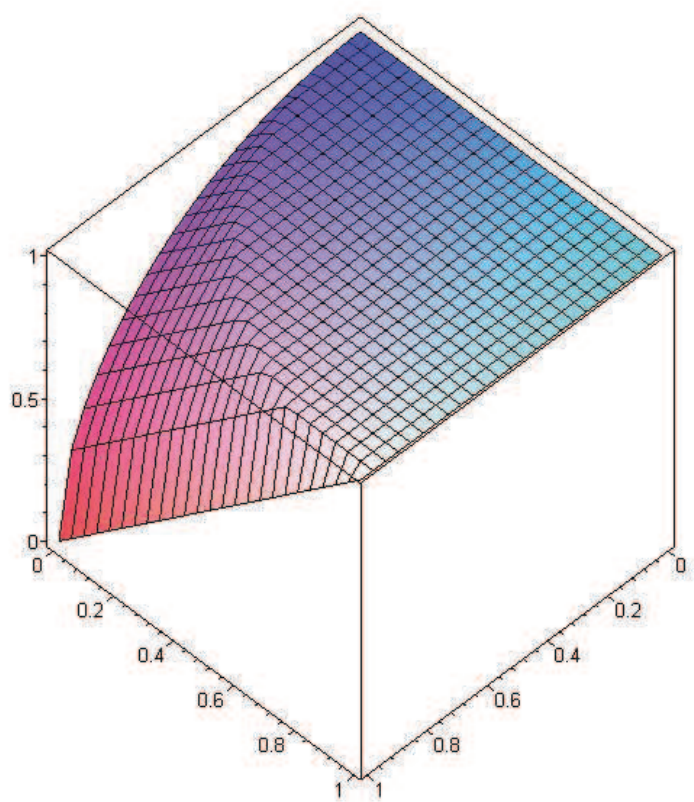

Fig. 3. The fuzzy implication $I_{4}$ in the proof of Proposition 5.1

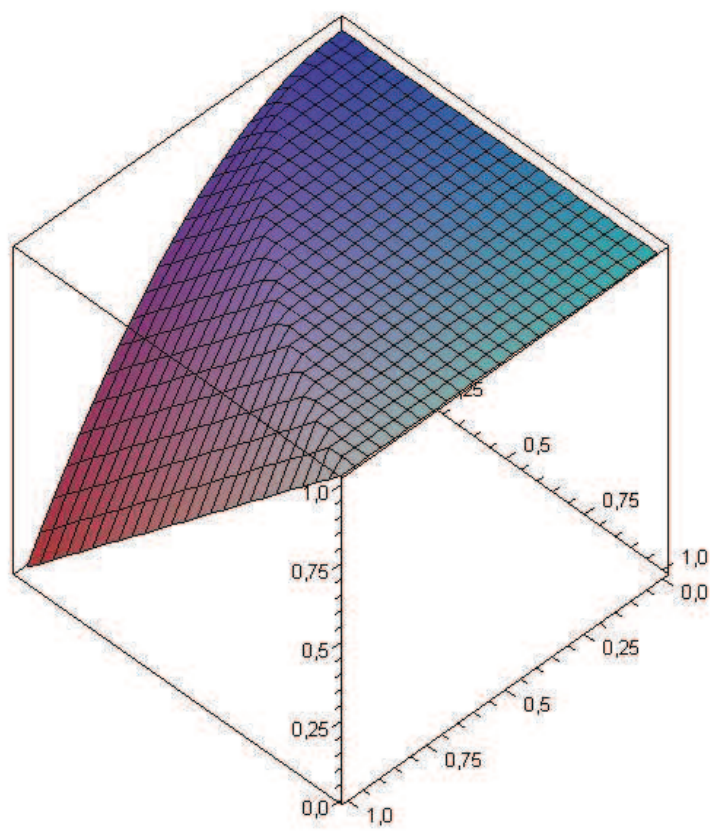

Fig. 4. The fuzzy implication $I_{5}$ in the proofs of Propositions 6.7 and 9.5 


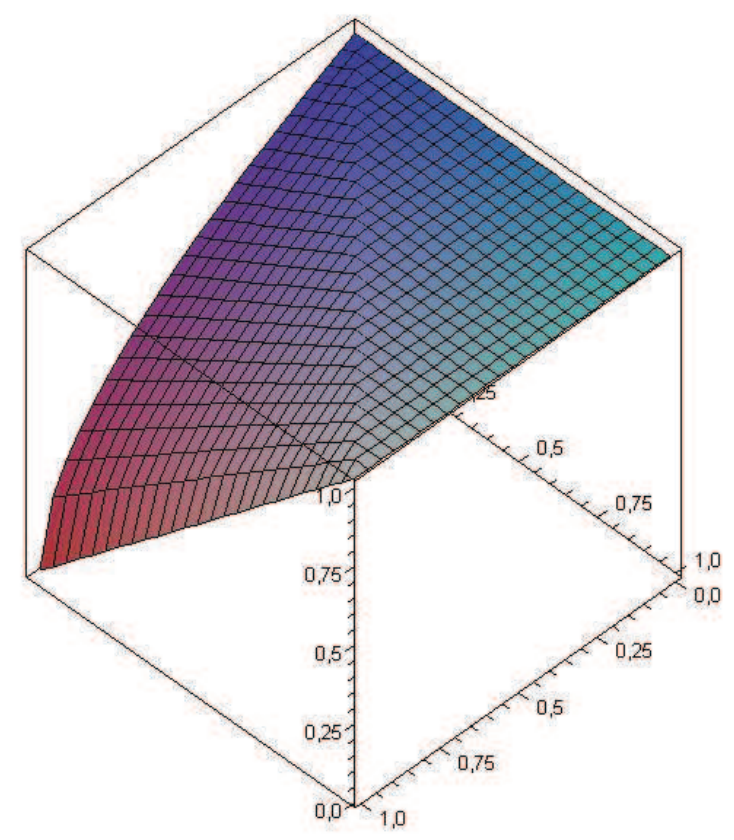

Fig. 5. The fuzzy implication $I_{6}$ in the proof of Proposition 6.8

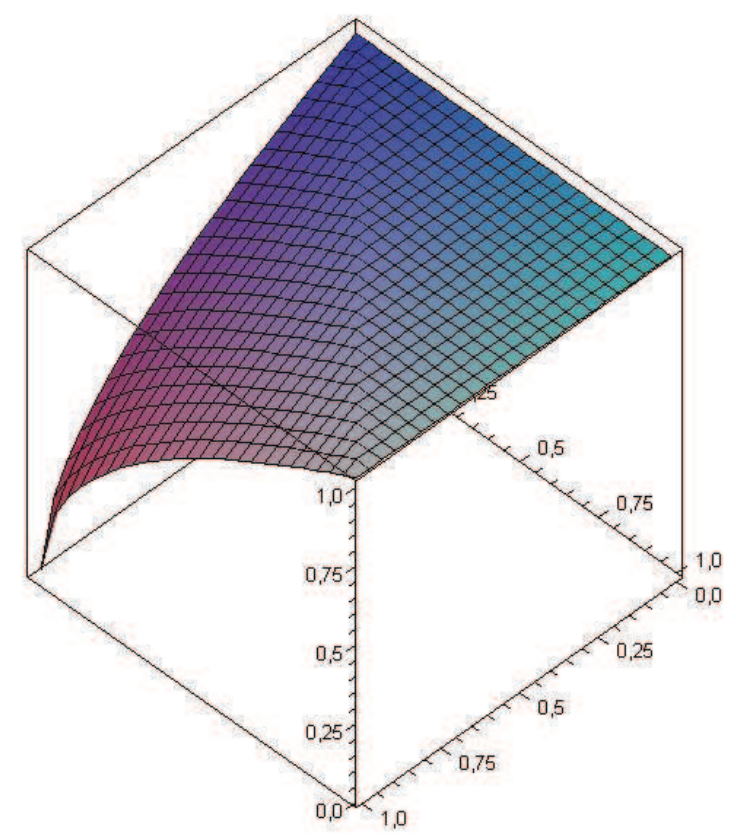

Fig. 6. The fuzzy implication $I_{7}$ in the proof of Proposition 6.10 


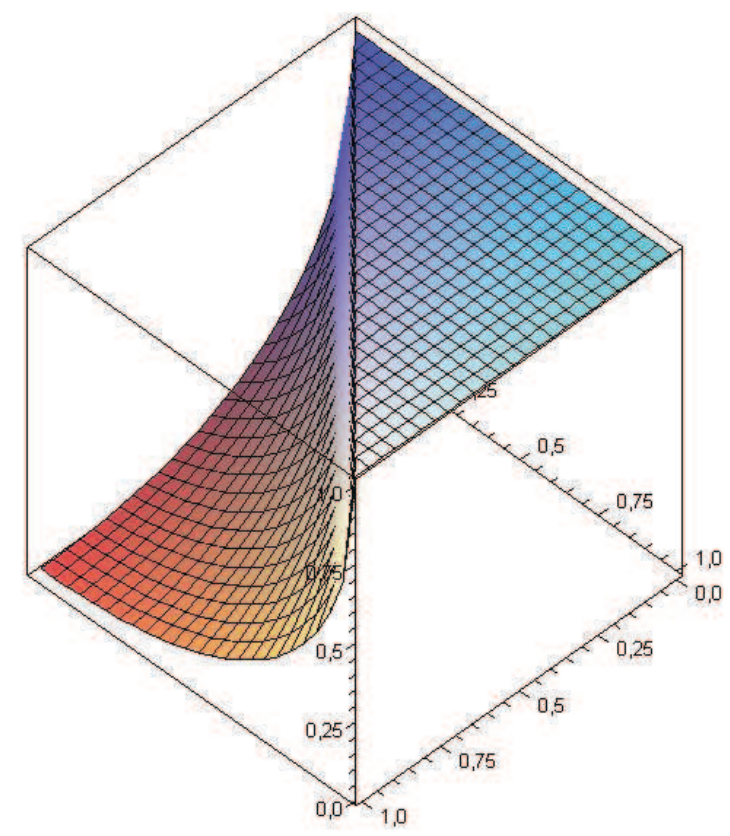

Fig. 7. The fuzzy implication $I_{9}$ in the proof of Proposition 7.7

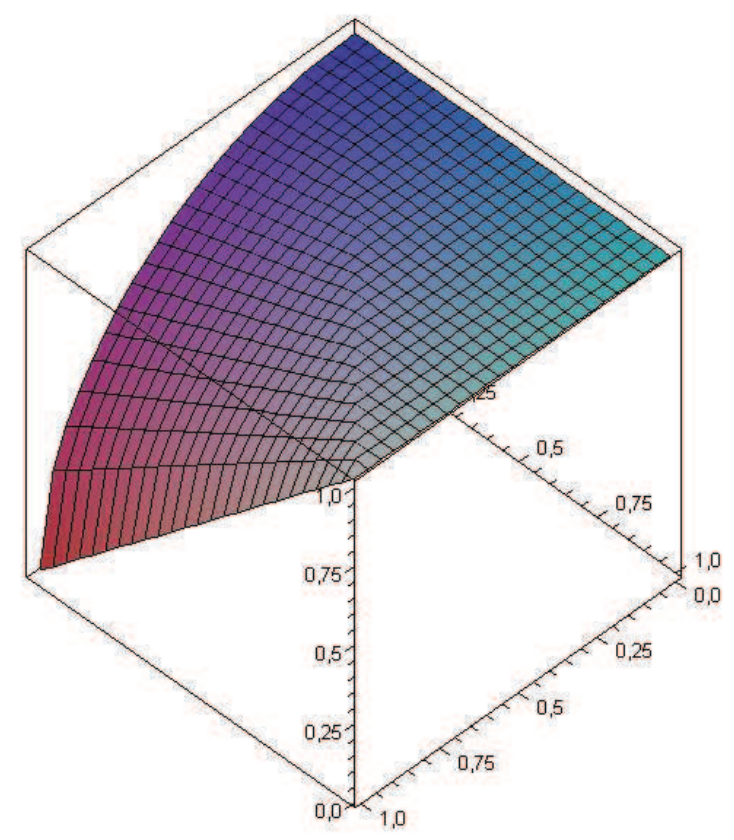

Fig. 8. The fuzzy implication $I_{10}$ in the proof of Proposition 9.6 


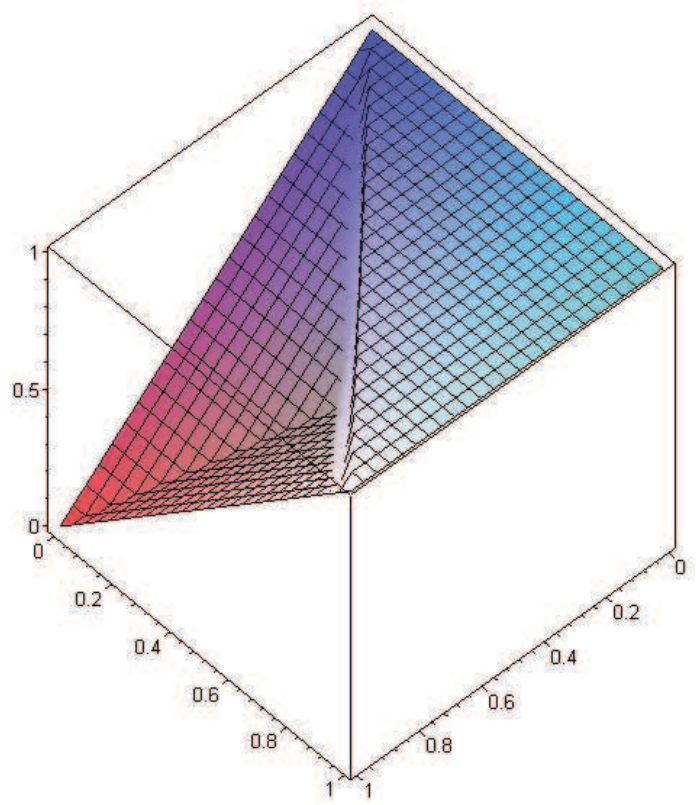

Fig. 9. The fuzzy implication $\left(I_{\min _{0}}\right)_{N_{0}}$ in the proof of Proposition 10.1 
Table 1

Summary of the interrelationships between the eight axioms

\begin{tabular}{|lc|}
\hline 1. & $\mathrm{EP} \wedge \mathrm{OP} \Rightarrow \mathrm{NT}$ \\
2. & $\mathrm{EP} \wedge \mathrm{SN} \Rightarrow \mathrm{NT}$ \\
3. & $\mathrm{EP} \wedge \mathrm{CO} \Rightarrow \mathrm{NT}$ \\
\hline \hline 4. & $\mathrm{NT} \wedge \mathrm{CP} \Rightarrow \mathrm{SN}$ \\
5. & $\mathrm{EP} \wedge \mathrm{OP} \wedge \mathrm{CP} \Rightarrow \mathrm{SN}$ \\
6. & $\mathrm{EP} \wedge \mathrm{OP} \wedge \mathrm{CO} \Rightarrow \mathrm{SN}$ \\
7. & $\mathrm{EP} \wedge \mathrm{CP} \wedge \mathrm{CO} \Rightarrow \mathrm{SN}$ \\
\hline \hline 8. & $\mathrm{NT} \Rightarrow \mathrm{CB}$ \\
9. & $\mathrm{EP} \wedge \mathrm{OP} \Rightarrow \mathrm{CB}$ \\
10. & $\mathrm{EP} \wedge \mathrm{SN} \Rightarrow \mathrm{CB}$ \\
11. & $\mathrm{EP} \wedge \mathrm{CO} \Rightarrow \mathrm{CB}$ \\
\hline \hline 12. & $\mathrm{OP} \Rightarrow \mathrm{ID}$ \\
\hline \hline 13. & $\mathrm{EP} \wedge \mathrm{OP} \wedge \mathrm{CO} \Rightarrow \mathrm{CP}$ \\
\hline 14. & $\mathrm{EP} \wedge \mathrm{SN} \Rightarrow \mathrm{CP}$ \\
\hline
\end{tabular}


Table 2

Summary of the examples of fuzzy implications satisfying the indicated axioms

\begin{tabular}{|c|c|c|c|c|c|c|c|c|}
\hline FI6 & FI7 & FI8 & FI9 & FI10 & FI11 & FI12 & FI13 & examples \\
NT & EP & OP & SN & CB & ID & CP & CO & \\
\hline $\mathrm{N}$ & $\mathrm{Y}$ & $\mathrm{N}$ & $\mathrm{N}$ & $\mathrm{Y}$ & $\mathrm{Y}$ & $\mathrm{Y}$ & $\mathrm{N}$ & $I_{1}$ \\
\hline $\mathrm{N}$ & $\mathrm{N}$ & $\mathrm{Y}$ & $\mathrm{Y}$ & $\mathrm{Y}$ & $\mathrm{Y}$ & $\mathrm{Y}$ & $\mathrm{Y}$ & $I_{2}$ \\
\hline $\mathrm{Y}$ & $\mathrm{N}$ & $\mathrm{Y}$ & $\mathrm{Y}$ & $\mathrm{Y}$ & $\mathrm{Y}$ & $\mathrm{Y}$ & $\mathrm{Y}$ & $I_{3}$ \\
\hline $\mathrm{Y}$ & $\mathrm{Y}$ & $\mathrm{N}$ & $\mathrm{Y}$ & $\mathrm{Y}$ & $\mathrm{Y}$ & $\mathrm{Y}$ & $\mathrm{Y}$ & $I_{4}$ \\
\hline $\mathrm{Y}$ & $\mathrm{Y}$ & $\mathrm{N}$ & $\mathrm{N}$ & $\mathrm{Y}$ & $\mathrm{Y}$ & $\mathrm{N}$ & $\mathrm{Y}$ & $I_{5}$ \\
\hline $\mathrm{Y}$ & $\mathrm{N}$ & $\mathrm{Y}$ & $\mathrm{N}$ & $\mathrm{Y}$ & $\mathrm{Y}$ & $\mathrm{N}$ & $\mathrm{Y}$ & $I_{6}$ \\
\hline $\mathrm{N}$ & $\mathrm{N}$ & $\mathrm{Y}$ & $\mathrm{N}$ & $\mathrm{Y}$ & $\mathrm{Y}$ & $\mathrm{Y}$ & $\mathrm{Y}$ & $I_{7}$ \\
\hline $\mathrm{N}$ & $\mathrm{Y}$ & $\mathrm{N}$ & $\mathrm{N}$ & $\mathrm{N}$ & $\mathrm{Y}$ & $\mathrm{Y}$ & $\mathrm{N}$ & $I_{8}$ \\
\hline $\mathrm{N}$ & $\mathrm{N}$ & $\mathrm{Y}$ & $\mathrm{Y}$ & $\mathrm{N}$ & $\mathrm{Y}$ & $\mathrm{Y}$ & $\mathrm{Y}$ & $I_{9}$ \\
\hline $\mathrm{Y}$ & $\mathrm{N}$ & $\mathrm{Y}$ & $\mathrm{Y}$ & $\mathrm{Y}$ & $\mathrm{Y}$ & $\mathrm{N}$ & $\mathrm{Y}$ & $I_{10}$ \\
\hline $\mathrm{Y}$ & $\mathrm{Y}$ & $\mathrm{Y}$ & $\mathrm{N}$ & $\mathrm{Y}$ & $\mathrm{Y}$ & $\mathrm{N}$ & $\mathrm{N}$ & $I_{G}$ \\
\hline $\mathrm{Y}$ & $\mathrm{Y}$ & $\mathrm{N}$ & $\mathrm{Y}$ & $\mathrm{Y}$ & $\mathrm{N}$ & $\mathrm{Y}$ & $\mathrm{Y}$ & $I_{K D}$ \\
\hline $\mathrm{Y}$ & $\mathrm{Y}$ & $\mathrm{Y}$ & $\mathrm{Y}$ & $\mathrm{Y}$ & $\mathrm{Y}$ & $\mathrm{Y}$ & $\mathrm{N}$ & $I_{\min _{0}}$ \\
\hline $\mathrm{Y}$ & $\mathrm{Y}$ & $\mathrm{Y}$ & $\mathrm{Y}$ & $\mathrm{Y}$ & $\mathrm{Y}$ & $\mathrm{Y}$ & $\mathrm{Y}$ & $I_{\mathrm{L}}$ \\
\hline
\end{tabular}

